\title{
Impact of everyday oral activities on the risk of bacteraemia is unclear
}

\author{
Abstracted from \\ Tomás I, Diz P, Tobías A, Scully C, Donos N. \\ Periodontal health status and bacteraemia from daily oral activities: systematic review/meta-analysis. \\ J Clin Periodontol 2012; 39: 213-228. Epub 2011 Sep 15. \\ Address for correspondence: I. Tomas, Special Needs Unit, \\ School of Medicine and Dentistry Santiago de Compostela University C./Entrerrios s/n15872 \\ Santiago de Compostela Spain. E-mail: inmaculada.tomas@usc.es
}

\section{Question: Do everyday oral activities result in bacteraemia?}

Data sources The Cochrane Library, Medline and Embase databases were searched as well as the references of identified articles and reviews. Study selection Studies were selected independently by two authors with disagreements being resolved by discussion. Only English language papers were included.

Data extraction and synthesis Data abstraction was conducted independently and meta-analysis conducted where appropriate. Results Twelve studies were included; seven on toothbrushing, one on dental flossing and four on chewing were included. Plaque and gingival indices were found to have a significant effect on the prevalence of bacteraemia following toothbrushing, with pooled odds ratios of 2.61 [95\% confidence interval $(\mathrm{Cl})=1.45-4.69$ ] and 2.77 $(95 \% \mathrm{Cl}=1.50-5.11)$ respectively. None of five studies on bacteraemia following dental flossing and chewing revealed a statistically significant association between oral hygiene, gingival or periodontal status and the development of bacteraemia.

Conclusions Meta-analysis showed that plaque accumulation and gingival inflammation scores significantly increased the prevalence of bacteraemia following toothbrushing. However, systematic review showed no relationship between oral hygiene, gingival and periodontal status and the development of B-chewing, and there is no evidence that gingival and periodontal health status affects B-flossing.

\section{Commentary}

The premise behind clinical guidelines recommending antibiotic prophylaxis for patients at risk of developing infective endocarditis (IE) or septic arthritis is that disruption of the integrity of the subgingival epithelium by routine dental procedures will lead to seeding of periodontal pathogens on susceptible target organs and tissues. This is purported to result in serious focal infections, and at first blush, seems biologically plausible. However, more recent evidence suggests that cumulative bacteraemia resulting from repetitive, everyday oral activities (EOA) - chewing, toothbrushing, dental flossing - are a more likely cause than a single dental procedure, ${ }^{1}$ particularly if high levels of virulent pathogens are present (ie in an unhealthy mouth).

This systematic review examines the evidence to support this hypothesis. It was well planned, being designed according to PRISMA guidelines. However, there are some gaps in the reporting. The inclusion criterion limiting studies to those with a minimal sample size of 11 subjects is not explained. As one of the benefits of metaanalysis is to overcome the limitation of individual studies with (potentially) small sample sizes by pooling results, it is not clear why this was criterion was chosen. In fact, the total number of patients included in the meta-analysis is not reported. In addition, the authors seem to misunderstand the purpose of planning to examine publication bias. They state that due to the small number of studies in the meta-analysis, examination of publication bias was not relevant. Rather, it was likely not possible with fewer than 10 included studies.

In order to standardise data, the authors used a threshold value of 1.5 for plaque (PI) and gingival (GI) indices, to compare those with good oral hygiene (PI = $0.0-1.5$ ) with those with poor oral hygiene (PI $>1.5)$ and those with good gingival health $(\mathrm{GI}=0.0-1.5)$ with those with poor gingival health $(\mathrm{GI}>1.5)$. While the mean value of the meta-analyses indicate the EOA may be more likely to cause bacteremia in those with poor oral hygiene and/or gingivitis, the lower limit of the confidence intervals includes 1.5. Thus, the answer is not as clear as the authors state. This is partly explained by the lack of agreement on the definitions of health, gingivitis and periodontitis, the lack of common measurement scales and the conversion of categorical scales (such as the gingival index) into interval data.

What is clear from this review is that there is no good evidence that everyday oral activities increase the likelihood of systemic bacteraemia. The good news is that this means it is unlikely that isolated dental procedures do so. Given the facts there is no evidence of cost-effectiveness associated with antibiotic prophylaxis; and the risks of doing so are high - development of antibiotic resistance and fatal anaphylaxis $^{1}$ - antibiotics should never be used as a preventive measure for IE. Yet, dental clinicians are routinely faced with demands by family doctors and medical specialists to prescribe antibiotics for their 'at-risk' patients. The findings of this systematic review speak most loudly to the failure of researchers to develop commonly accepted protocols, definitions of disease and outcome measurements, so that we have clear evidence with which to educate our medical colleagues on this issue - for the benefit of all our patients.

Debora Matthews

Department of Dental Clinical Sciences, Dalhousie University, Halifax, Nova Scotia, Canada

1. National Institute for Health and Clinical Excellence. Prophylaxis against infective endocarditis. Antimicrobial prophylaxis against infective endocarditis in adults and children undergoing interventional procedures.http://www.nice.org.uk/CG064. (Accessed 30 July 2012)

Evidence-Based Dentistry (2012) 13, 80. doi:10.1038/sj.ebd.6400875 